\title{
Editorial
}

\section{Analytic and Harmonic Univalent Functions}

\author{
V. Ravichandran, ${ }^{1}$ Om P. Ahuja, ${ }^{2}$ and Rosihan $\mathrm{M} . \mathrm{Ali}^{3}$ \\ ${ }^{1}$ Department of Mathematics, University of Delhi, Delhi 110 007, India \\ ${ }^{2}$ Department of Mathematical Sciences, Kent State University, Burton, OH 44021, USA \\ ${ }^{3}$ School of Mathematical Sciences, Universiti Sains Malaysia, 11800 USM, Penang, Malaysia \\ Correspondence should be addressed to V. Ravichandran; vravi68@gmail.com
}

Received 14 October 2014; Accepted 14 October 2014; Published 22 December 2014

Copyright (C) 2014 V. Ravichandran et al. This is an open access article distributed under the Creative Commons Attribution License, which permits unrestricted use, distribution, and reproduction in any medium, provided the original work is properly cited.

Studies on analytic univalent functions became the focus of intense research with the Bieberbach conjecture posed in 1916 concerning the size of the moduli of the Taylor coefficients of these functions. In efforts towards its resolution, the conjecture inspired the development of several ingeniously different mathematical techniques with powerful influence. These techniques include Lowner's parametric representation method, the area method, Grunsky inequalities, and methods of variations. Despite the fact that the conjecture was affirmatively settled by de Branges in 1985, complex function theory continued to remain a highly active relevant area of research.

Closely connected are harmonic univalent mappings, which are widely known to have a wealth of applications. They arise in the modelling of many physical problems, such as in the study of fluid dynamics and elasticity problems, in the approximation theory of plates subjected to normal loading, and in the investigations of Stokes flow in the engineering and biological transport phenomena. Harmonic mappings are also important to differential geometers because these maps provide isothermal (or conformal) parameters for minimal surfaces. Indeed various properties of minimal surfaces such as the Gauss curvature are studied more effectively through planar harmonic mappings.

Although a harmonic map provides a natural generalization to studies on analytic univalent functions, surprisingly it fails to capture the interest of function theorists for quite a period of time. The defining moment came with the seminal paper by Clunie and Sheil-Small in 1984. They introduced complex analytic approach in their studies and succeeded in finding viable analogues of the classical growth and distortion theorems, covering theorems, and coefficient estimates in the general setting of planar harmonic mappings. Although there have been substantial steps forward in the studies of harmonic mappings, yet many fundamental questions and conjectures remain unresolved. There is a great expectation that the "harmonic Koebe function" will play the extremal role in many of these problems, much akin to the role played by the Koebe function in the classical theory of analytic univalent functions.

This special issue aims to disseminate recent advances in the studies of complex function theory, harmonic univalent functions, and their connections to produce deeper insights and better understanding. These are crystallized in the form of original research articles or expository survey papers.

The response to this special issue was beyond our expectations. Forty papers were received in several areas of research fields in analytic and harmonic univalent functions. All submitted papers went through a rigorous scrutiny of two or three peer-reviewed processes. Based on the reviewers' reports and editors' reviews, thirteen original research articles were selected for inclusion in the special issue.

New concepts and techniques in the theory of first, second, and third order differential subordination and superordination for analytic functions (as well as nonanalytic functions) were introduced. These can be found in the three papers entitled "Third-order differential subordination and superordination results for meromorphically multivalent functions associated with the Liu-Srivastava operator" (H. Tang et al.), "Differential subordinations for nonanalytic functions" (G. I. Oros and G. Oros), and "Differential subordination 
results for analytic functions in the upper half-plane" $(\mathrm{H}$. Tang et al.). The paper "Meromorphic solutions of some algebraic differential equations" (J. Lin et al.) provides estimates on the growth order of meromorphic solutions of certain algebraic differential equations by means of the normal family theory. The paper "A note on entire functions that share two small functions" by J.-F. Chen investigates a close linear relationship between a non-constant entire function under certain conditions and its first derivative.

The paper "Upper bound of second Hankel determinant for certain subclasses of analytic functions" (M.-S. Liu et al.) summarizes works done in the area of the Hankel determinant for univalent functions, while the paper "Initial coefficients of biunivalent functions" (S. K. Lee et al.) gives estimates on the initial coefficients of the Taylor coefficients of these functions. The paper "Some connections between class $\mathcal{U}$ - and $\alpha$-convex functions" (E. Aliaga and $\mathrm{N}$. Tuneski) gives sufficient conditions for an $\alpha$-convex function to be in a specific class, while the paper "Starlikeness offunctions defined by third-order differential inequalities and integral operators" (R. Chandrashekar et al.) determines sufficient conditions for analytic functions satisfying certain third-order differential inequalities to be starlike.

Four papers in this issue deal with univalent harmonic mappings. These papers are "Radius constants for functions with the prescribed coefficient bounds" (O. P. Ahuja et al.), "On certain subclass of harmonic starlike functions" (A. Y. Lashin), "A family of minimal surfaces and univalent planar harmonic mappings" (M. Dorff and S. Muir), and "Landautype theorems for certain biharmonic mappings" (M.-S. Liu et al.). The first of these four papers, "Radius constants for functions with the prescribed coefficient bounds," establishes a coefficient inequality for sense-preserving harmonic functions to compute the bounds for the radius of univalence and radius of full starlikeness (and convexity) of positive order for functions with prescribed coefficient bound on the analytic part; the second one, "On certain subclass of harmonic starlike functions," discusses the geometric properties for a new class of harmonic univalent functions; the third one, "A family of minimal surfaces and univalent planar harmonic mappings," presents a two-parameter family of minimal surfaces constructed by lifting a family of planar harmonic mappings, while the fourth one, "Landau-type theorems for certain biharmonic mappings," proves the Landau-type theorems for biharmonic mappings connected with a linear complex operator.

We hope that the papers in this special issue will help enrich our readers and stimulate further research.

\section{Acknowledgment}

Finally we take this opportunity to express our heartfelt gratitude to all contributing authors and to the reviewers for ensuring the high standards of the issue.

V. Ravichandran

Om P. Ahuja

Rosihan M. Ali 


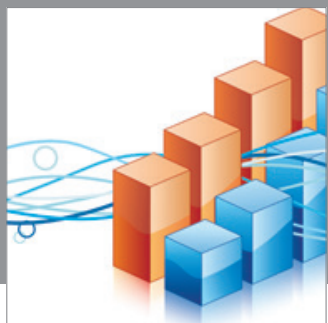

Advances in

Operations Research

mansans

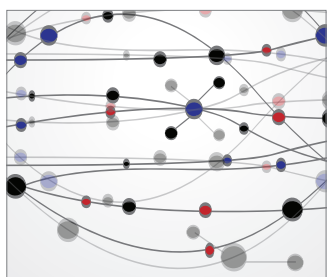

The Scientific World Journal
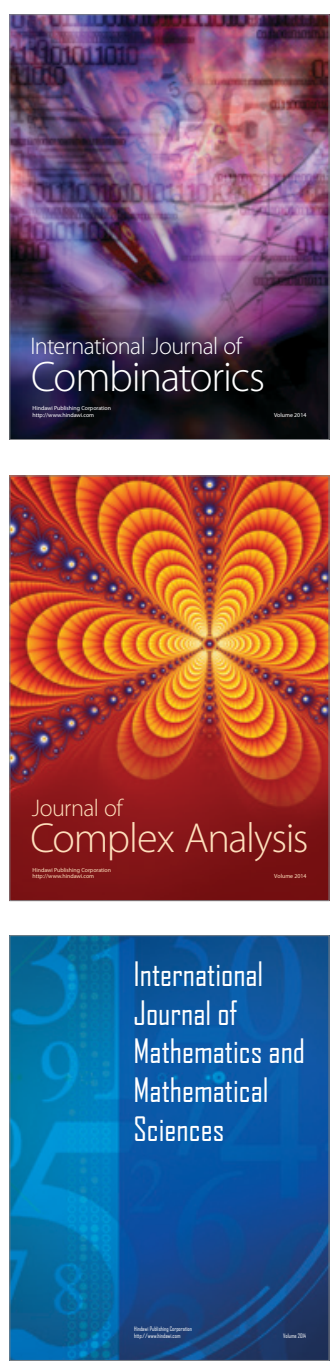
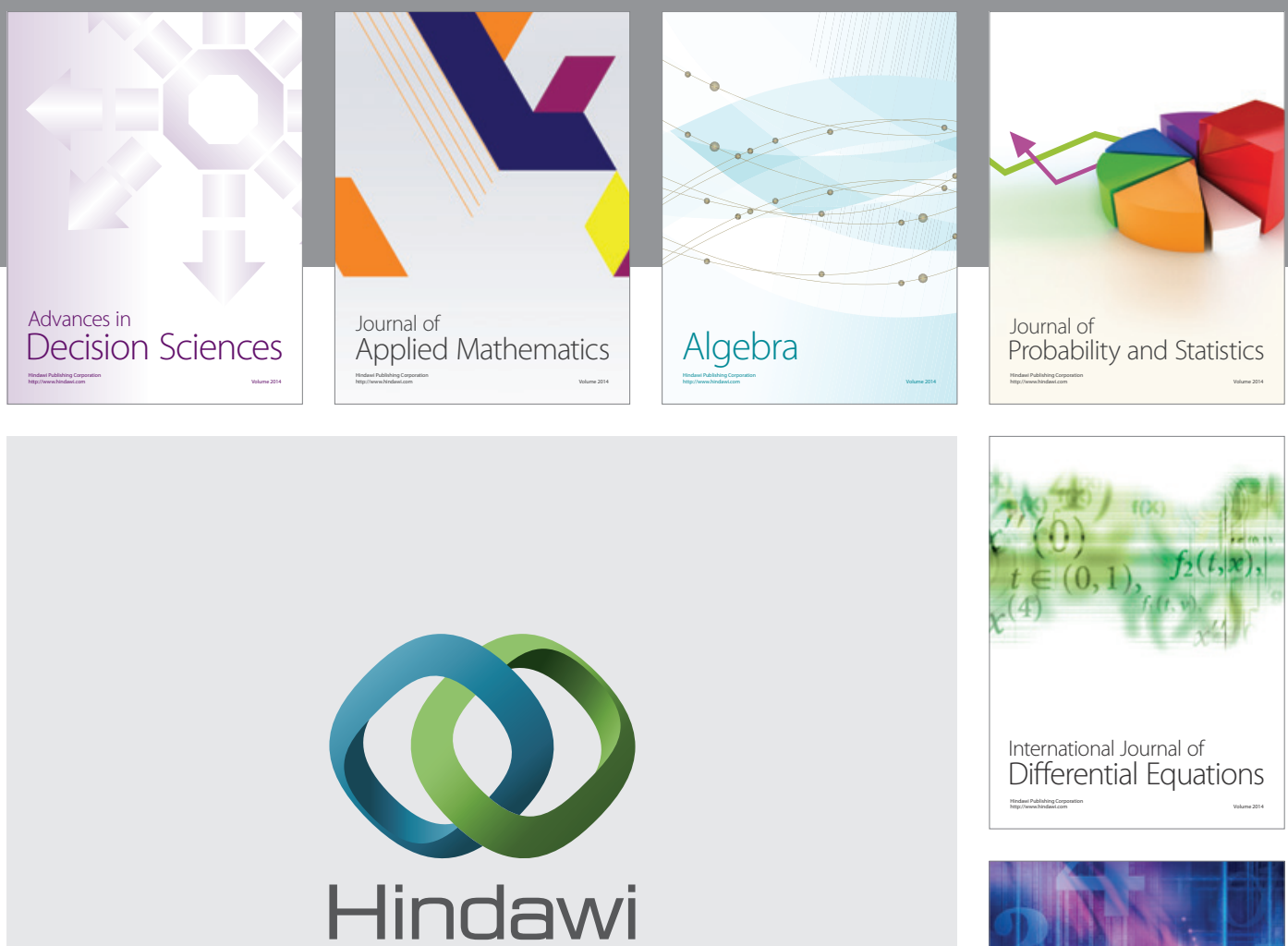

Submit your manuscripts at http://www.hindawi.com
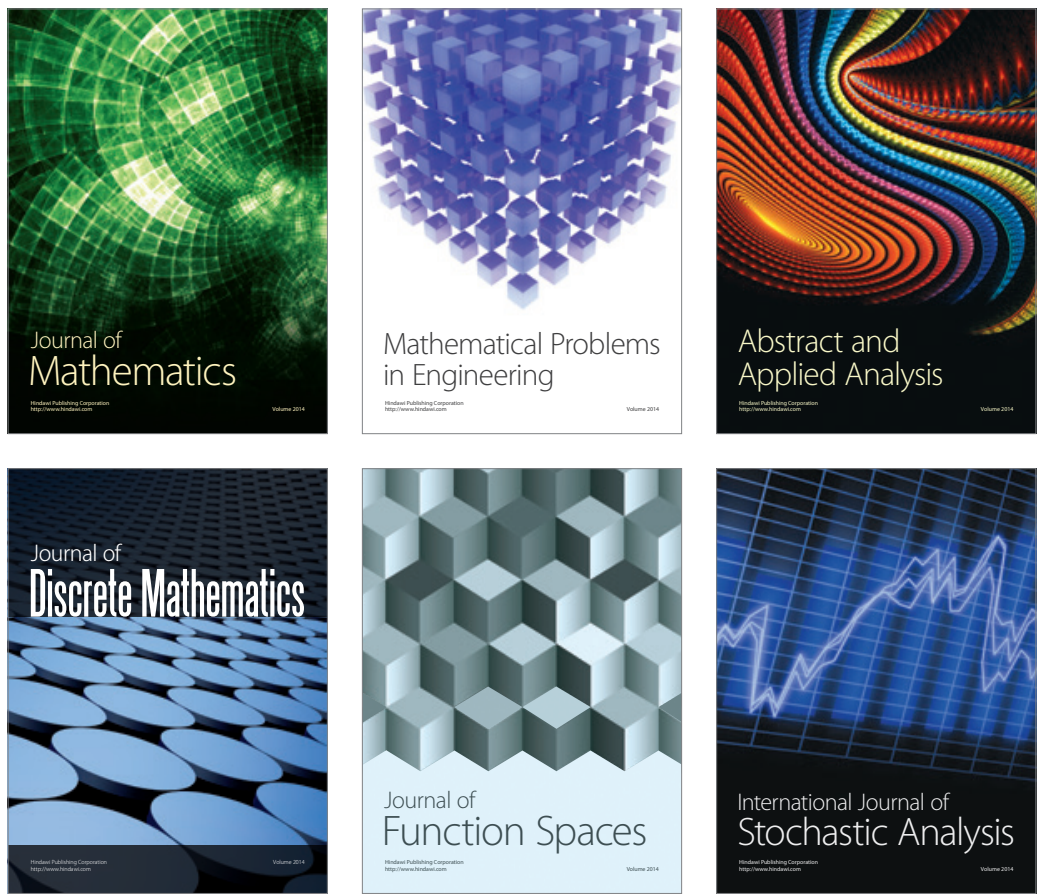

Journal of

Function Spaces

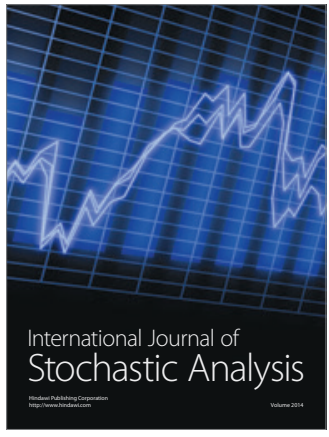

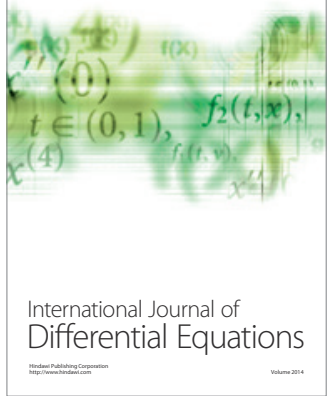
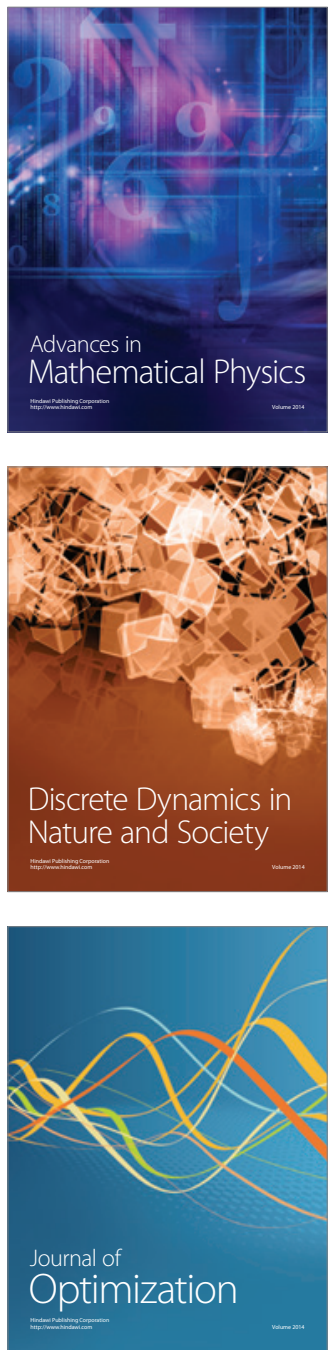